\title{
Lipid-liver crosstalk in health and disease
}

\begin{abstract}
The liver is a major metabolic organ, essentially controlling glucose and fat metabolism. Because lipids are water-insoluble, they need to be transported in the circulation in association with proteins in the form of lipoproteins. These lipoproteins play a key role in the absorption and transport of dietary lipids by the small intestine, in the transport of lipids from the liver to peripheral tissues, and in the transport of lipids from peripheral tissues to the liver and intestine. Upon feeding, energy is provided by glycolysis and the unused glucose is stored as glycogen in the liver. Excess glucose is used to synthesize fatty acids through de novo lipogenesis. Fatty acids are incorporated into triacylglycerol, phospholipids, or cholesterol esters in hepatocytes. These complex lipids are stored in lipid droplets or secreted into the circulation as very low-density lipoprotein particles. Upon fasting, after consumption of stored glycogen, the liver secretes glucose through gluconeogenesis. Fasting also promotes lipolysis in adipose tissue, resulting in release of free fatty acids which are metabolized in hepatic mitochondria though beta-oxidation for energy production, while excess fatty acids are stored by the liver leading to hepatic steatosis. Disturbances in lipid metabolism, as in alcoholic and non-alcoholic steatohepatitis, obesity and diabetes, will affect liver performance and function. Likewise, disturbed liver functions by acute or chronic liver disease, as in viral hepatitis, will affect lipid homeostasis. This may carry an increased risk of atherosclerosis and ischemic heart disease which may endanger life. Dietary restriction or fasting was found to have a positive impact on restoring lipid homeostasis leading to improved quality of life.
\end{abstract}

Keywords: lipids, lipoproteins, dyslipidemia, atherosclerosis, fatty liver disease
Volume 2 Issue I - 2016

\author{
Mostafa Mohamed Sira,' Ahmed Mostafa \\ Sira ${ }^{2}$ \\ 'Department of Pediatric Hepatology, Menofiya University, Egypt \\ ${ }^{2}$ Department of Medicine and Surgery, Second level Medical \\ College, Egypt
}

Correspondence: Mostafa M Sira, Department of Pediatric Hepatology, National Liver Institute, Menofiya University, 325 I I Shebin El-koom, Menofiya, Egypt, Tel +2 048222 2740, Fax +204 8223 4586,Email msira@liver-eg.org

Received: November 27, 2015 | Published: January 27, 2016
Abbreviations: NAFLD, non-alcoholic fatty liver disease; TG, triglycerides; VLDL, very low density lipoprotein; IDL, intermediate density lipoprotein; LDL, low density lipoprotein; HDL, high density lipoprotein; FFA, free fatty acid; CYP4A, cytochrome p4a; NASH, non-alcoholic steatohepatitis; AFLD, alcoholic fatty liver disease

\section{Introduction}

Nutrition and the liver are interrelated in many ways. The liver plays a key role in converting food into the chemicals essential for life and serves several important metabolic functions in handling nutrients. Fatty liver diseases are linked to poor nutrition, obesity and sedentary lifestyle. In addition to obesity, fatty liver disease has also been linked to other risk factors, including insulin resistance, hyperlipidaemia, high blood pressure, and type II diabetes. ${ }^{1}$

Non-alcoholic fatty liver disease (NAFLD) is the most common cause of liver disease worldwide, with a prevalence of $20 \%-40 \%$ in Western populations. ${ }^{2}$ In Europe, the prevalence of NAFLD varies between $20-30 \%{ }^{3}$ The prevalence increases to $58 \%$ in overweight individuals and can be as high as $98 \%$ in non-diabetic obese individuals. ${ }^{4}$ The problem is also prevailing in the developing counties $^{5}$ and is considered a global epidemic., ${ }^{6,7}$

The current review provides a comprehensive exploration of the lipid-liver crosstalk. This will help for the early anticipation and management of such metabolic disturbance by treating the offending factor and/or manipulating the caloric intake. In such way, lipid-liver homeostasis can be restored. This will reduce the morbidity, and improve quality of life.

\section{Lipid transport and lipoprotein pathway}

Because lipids such as cholesterol and triglycerides (TG) are water-insoluble, these lipids need to be transported in the circulation in association with proteins in the form of lipoproteins. Large quantities of fatty acids from diet must be transported as TG to avoid toxicity. These lipoproteins play a key role in the absorption and transport of dietary lipids by the small intestine, in the transport of lipids from the liver to peripheral tissues, and in the transport of lipids from peripheral tissues to the liver and intestine. Lipoproteins are complex particles that have a central hydrophobic core of non-polar lipids, primarily cholesterol esters and TG. This hydrophobic core is surrounded by a hydrophilic membrane consisting of phospholipids, free cholesterol, and apolipoproteins. Plasma lipoproteins vary according to size, lipid composition, and apolipoproteins and hence the function also varies (Table 1). ${ }^{8}$

The exogenous lipoprotein pathway starts with the incorporation of dietary lipids into chylomicrons in the intestine. In the circulation the TG carried in chylomicrons are metabolized in muscle and adipose tissue by lipoprotein lipase and then, chylomicron remnants are formed. The chylomicron remnants are then taken up by the liver. The endogenous lipoprotein pathway begins in the liver with the formation of very low density lipoprotein (VLDL). The TG carried in VLDL's are metabolized in muscle and adipose tissue by lipoprotein lipase and intermediate density lipoprotein (IDL) are formed. The IDL are further metabolized with more consumption of its TG leading to the formation of low density lipoprotein (LDL), which are taken up by the LDL receptors in numerous tissues including the liver as the predominant site of uptake. Reverse cholesterol transport begins with the formation of nascent high density lipoprotein (HDL) by the liver and intestine. These nascent HDL can then acquire cholesterol and phospholipids from peripheral tissues and blood vessels. The HDL then transports the cholesterol to the liver. Cholesterol efflux from macrophages and blood vessels to HDL plays an important role in protecting from the development of atherosclerosis (Table 1). ${ }^{9}$ 
Table I Major lipoprotein classes found in plasma.APO, apolipoproteins; HDL, high density lipoprotein; IDL, intermediate density lipoprotein; LDL, low density lipoprotein;VLDL, very low density lipoprotein. From Fox $2016^{86}$ with modifications

\begin{tabular}{|c|c|c|c|c|c|}
\hline Lipoprotein & Origin & Destination & Major Lipids & $\begin{array}{l}\text { Major } \\
\text { Apolipoproteins }\end{array}$ & Functions \\
\hline Chylomicrons & Intestine & $\begin{array}{l}\text { Through lymphatics to } \\
\text { blood to many organs } \\
\text { and tissues }\end{array}$ & Triglycerides & $\begin{array}{l}\text { Apo B-48,Apo C,Apo } \\
\text { E,Apo A-I,A-II,A-IV }\end{array}$ & $\begin{array}{l}\text { Deliver lipids of dietary } \\
\text { lipids origin to body cells }\end{array}$ \\
\hline VLDL & Liver & Many organs and tissues & $\begin{array}{l}\text { Triglycerides } \\
\text { Cholesterol }\end{array}$ & $\begin{array}{l}\text { Apo B-100,Apo E, } \\
\text { Apo C }\end{array}$ & $\begin{array}{l}\text { Deliver endogenously pro- } \\
\text { duced triglycerides to body } \\
\text { cells }\end{array}$ \\
\hline IDL & $\begin{array}{l}\text { Intravascular } \\
\text { removal of } \\
\text { Triglycerides } \\
\text { from VLDL }\end{array}$ & Blood vessels and liver & $\begin{array}{l}\text { Triglycerides } \\
\text { Cholesterol }\end{array}$ & $\begin{array}{l}\text { Apo B-100,Apo E, } \\
\text { Apo C }\end{array}$ & $\begin{array}{l}\text { Deliver part of endogenous- } \\
\text { ly produced triglycerides to } \\
\text { body cells and the remaining } \\
\text { part is carried back to the } \\
\text { liver }\end{array}$ \\
\hline LDL & $\begin{array}{l}\text { Intravascular } \\
\text { removal of } \\
\text { Triglycerides } \\
\text { from IDL }\end{array}$ & Blood vessels and liver & Cholesterol & Apo B- 100 & $\begin{array}{l}\text { Deliver endogenously pro- } \\
\text { duced cholesterol to various } \\
\text { organs and blood vessels }\end{array}$ \\
\hline HDL & $\begin{array}{l}\text { Liver and } \\
\text { intestine }\end{array}$ & $\begin{array}{l}\text { Liver and steroid } \\
\text { hormone producing } \\
\text { glands (testis, ovaries, } \\
\text { adrenal medulla) }\end{array}$ & $\begin{array}{l}\text { Cholesterol } \\
\text { Phospholipids }\end{array}$ & $\begin{array}{l}\text { Apo A-I,Apo A-II,Apo } \\
\text { C,Apo E }\end{array}$ & $\begin{array}{l}\text { - Remove cholesterol from } \\
\text { peripheral tissues and blood } \\
\text { vessels } \\
\text {-Deliver cholesterol to hor- } \\
\text { mone producing glands } \\
\text {-Deliver triglycerdies from } \\
\text { VLDL and LDL to the liver }\end{array}$ \\
\hline
\end{tabular}

\section{Lipid handling by the liver}

The liver is a key player in the whole body energy homeostasis by its ability to metabolize glucose and fatty acids. When energy intake is abundant, cells preferentially burn carbohydrates to generate adenosine tri-phosphate and excess glucose, after replenishing glycogen stores, is converted to fatty acids (lipogenesis) for use in the synthesis and storage of TG in adipose tissue. ${ }^{10}$ Although adipose tissue functions essentially as a limitless reservoir to accumulate TG, the liver is also able to store significant quantities of lipids in conditions associated with prolonged excess energy consumption or impaired fatty acid metabolism manifesting as steatosis. During fasting, when glucose availability and insulin levels are low, there is a depletion of hepatic glycogen stores and a reduction in fatty acid production. Under these conditions, TG stored in adipose tissues are hydrolyzed to free fatty acids (FFA) and mobilized into plasma to reach the liver. In the liver, they undergo oxidation and converted to ketone bodies to be used as fuel by extrahepatic tissues. ${ }^{11}$

Disturbances in fatty acid oxidation will lead to excess lipid storage in the liver. Fatty acid oxidation is roughly proportional to the plasma concentration of FFA released from adipose tissue. Fatty acid mobilization is stimulated by glucagon and inhibited by insulin. Oxidation of fatty acids occurs in three sub cellular organelles, with oxidation confined to mitochondria and peroxisomes and the cytochrome P4A (CYP4A)-catalyzed omega-oxidation occurring in the endoplasmic reticulum. ${ }^{10}$

Mitochondrial oxidation is primarily involved in the oxidation of short-chain (less than 8 carbons length), medium-chain (8-12carbons length), and long-chain (12- 20 carbon length) fatty acids, and this process provides energy to cellular processes. $^{12}$ Mitochondrial oxidation results in shortening of fatty acids progressively into acetyl-Co A subunits, which either condenses into ketone bodies that is oxidized to produce energy for extrahepatic tissues, especially during starvation, or enter into the tri carboxylic acid cycle for further oxidation with production of adenosine tri-phosphate. ${ }^{13}$

Paroxysmal oxidation is concerned exclusively with the metabolism of less abundant and relatively more toxic very-long-chain fatty acids as (containing more than 20carbons), 2-methyl-branched fatty acids, di carboxylic acids, prostanoids, and $\mathrm{C} 27$ bile acid intermediates. Very-long-chain fatty acids are not processed by the mitochondrial oxidation system, and they require peroxisomal oxidation to shorten the chain length for further completion of oxidation in mitochondria. Long-chain di carboxylic acids generated by the microsomal oxidation of fatty acids are metabolized by the peroxisomal oxidation system. ${ }^{14}$ Di carboxylic acids are generally more toxic than very-longchain fatty acids and are known to inhibit the mitochondrial fatty acid oxidation system. An effective peroxisomal oxidation system is needed to protect the liver from the harmful effects of di carboxylic and other toxic fatty acids. ${ }^{11,15}$

Fatty acids are also oxidized by the microsomal oxidation system by CYP4A enzymes capable of hydroxylating saturated and unsaturated fatty acids. The first step in microsomal fatty acid oxidation is hydroxylation in the endoplasmic reticulum, and the resulting hydroxy fatty acid is then dehydrogenated to a di carboxylic acid in the cytosol. Di carboxylic acids are then converted to di carboxyl-Co A's for oxidation by the classical oxidation pathway. ${ }^{12}$ 


\section{Disturbed lipid metabolism and secondary liver affection}

Hepatic steatosis, or the accumulation of significant amounts of TG in hepatocytes, was long thought to be mainly a symptom of alcoholic drinking leading to liver disease. In recent years, steatosis has been found in the absence of alcohol intake and led to the definition of a new category of disorders ranging from nonalcoholic fatty liver (NAFL) to nonalcoholic steatohepatitis (NASH) ${ }^{16}$ Hepatic steatosis may progress to steatohepatitis which leads to fibrosis and cirrhosis in late stages. This progression may be due to damage caused by lipid peroxidation and the production of reactive oxygen species. Excess lipid peroxidation is dependent on excess substrate, which is the stored lipid in hepatocytes as TG. ${ }^{17}$

Sources of increased lipid, TG, content in hepatic steatosis include excess dietary TG associated with overeating that reach the liver as chylomicron particles from the intestine, increased TG synthesis in the liver from fatty acids as de novo lipogenesis, excess fatty acid influx into the liver from lipolysis of adipose tissue in obese and insulin resistant states and subsequent conversion to TG, diminished export of lipids from the liver in VLDL, and fatty acids oxidation defect. High insulin inhibits hepatic glucose production, increases hepatic glucose uptake, and enhances lipogenesis in the liver. Generally, conditions encouraging fatty acid influx into the liver, their de novo synthesis, and conversion to TG and/or oxidation to generate adenosine triphosphate contribute to disturbances in hepatic lipid homeostasis. ${ }^{18,19}$

Lipids arrive at the hepatocyte surface in a number of forms. Both FFA and lipoprotein particles provide lipids to the liver. Although some lipoprotein particles are taken up by receptor-mediated endocytosis, in others, the TG may be broken down by hepatic lipase, to produce FFA, which cross the hepatocyte membrane by a combination of facilitated transport and diffusion. ${ }^{17}$ In hepatocytes, the FFA may be converted to TG or oxidized as fuel. Some may be used in the synthesis of phospholipids and mediators such as prostaglandins and leukotrienes and parts of the carbon skeletons may be used to synthesize glucose, cholesterol, and other compounds. FFA may also be converted to TG, which can be used for production of VLDL particles for lipid export from the liver. ${ }^{20}$

Excess FFA may be transported out of cells by the same mechanisms that allow for uptake, but this is likely a minor fraction, because FFA are modified by fatty acyl-Co A synthetases immediately after entry into cells and became unavailable for transport or diffusion. The majority of excess FFA is likely converted to TG, and stored in hepatocytes. ${ }^{21}$ The storage capacity of hepatocytes can be increased by increased diffusion, such as by increased cell surface area or alterations in membrane composition, or by increasing the number of transporter molecules. If a transporter be up regulated inappropriately, it could lead to increased hepatocyte FFA uptake and contribute to steatosis and subsequent clinical problems. ${ }^{22}$

Excessive fat in the diet, will lead to increased plasma concentrations of TG and FFA. The increased FFA concentration will allow for increased uptake into hepatocytes. ${ }^{17}$ Furthermore, because carbohydrates and proteins can serve as sources for synthesis of fatty acids, excess caloric intake results in increased adiposity, and eventual obesity. Hepatic steatosis can occur in the presence of obesity, especially in those with type 2diabetes and the metabolic syndrome. Type 2diabetes usually occurs in association with obesity but with the added problem of insulin resistance. Peripheral tissues fail to respond to insulin in a normal manner, leading to lowered utilization of glucose as an energy source. ${ }^{23}$
Obesity and obesity-associated fatty liver disease (FLD) are becoming global health problems in adults as well as children. When caloric intake far exceeds the daily needs, the unused energy is conserved in the form of TG in adipose tissue, leading to obesity. Obesity-associated insulin resistance appears to serve as a pathogenic event responsible for the metabolic syndrome comprising type 2diabetes mellitus, dyslipidemia, atherosclerosis, hypertension, and hepatic steatosis progressing to FLD. Insulin is normally a stimulus for adipose tissue to take up more FFA from lipoproteins and to export less FFA. Insulin resistance depresses both of this functions. ${ }^{17}$

The result of insulin resistance in adipocytes is lowered conversion of lipoprotein TG to FFA for uptake and increased breakdown of stored TG for export. This might be beneficial to the adipocyte, as it protects from storing excessive amounts of fat. Also, increased lipolysis leads to increased FFA export into the circulation. This will cause some FFA to be returned to adipocytes, but the plasma FFA concentration will continue to rise with increase in FFA flux across the hepatocyte membrane..$^{23}$

Conditions such as lipodystrophy, where adipose depots are severely reduced, or absent, prevent the normal storage of fat in adipocytes. Therefore, the plasma will have elevated levels of FFA and TG and the liver will be one organ capable of removing them from circulation. In such case, any abnormality that leads to elevated plasma FFA and TG will predispose to hepatic steatosis, including rapid weight loss. ${ }^{17}$

Hepatic steatosis is encountered in about $20-35 \%$ of the general adult population in the United States with about $10 \%$ of these advancing toward NAFLD, ${ }^{24}$ while, the prevalence of steatosis in obese individuals is about $75 \%$, and nearly $35 \%$ or more of these develop NAFLD. ${ }^{25,26}$ FLD, whether it is alcoholic fatty liver disease (AFLD) or NAFLD, encompasses a morphological spectrum consisting of hepatic steatosis and steatohepatitis, whether alcoholic steatohepatitis (ASH) or NASH, that may progress toward the development of cirrhosis and hepatocellular carcinoma. ${ }^{19,24,26,27}$ NAFLD is currently the most common form of chronic liver disease in both children and adults and threatens to become a serious public health problem. ${ }^{28}$ It is estimated that one in three adults and one in 10 children or adolescents in the United States have NAFLD. ${ }^{26,29}$

Hepatic steatosis is considered benign in its bland form, reversible, and to a large extent non-progressive if the underlying cause is properly dealt with. Progression to steatohepatitis, ASH or NASH, is influenced by the persistence and severity of the cause of hepatic steatosis. Steatohepatitis in ASH and NASH represents an association of macro-vesicular steatosis with necro-inflammatory features such as ballooning hepatocytes, apoptotic cells, and infiltration of inflammatory cells. The extent of the inflammatory response varies considerably and does not always correlate with the degree of steatosis. ${ }^{11}$

The pathogenesis of ASH and NASH is multifactorial and includes several overlapping events such as reactive oxygen species, gut-derived endotoxins and adipocytokines such as tumor necrosis factor alpha and other cytokines. The precipitating or inciting event may be directly related to rupture or apoptosis of markedly steatotic hepatocytes and the release of TG and toxic fatty acids which act as an inducer of fatty acid oxidation systems that generate reactive oxygen species resulting in oxidative stress. ${ }^{14}$ Oxidative stress causes the release of several cytokines including tumor necrosis factor alpha, transforming growth factor and interleukins by Kupffer cells. ${ }^{30}$ 
Other causes of NAFLD include Parenteral nutrition, gastric bypass surgery, and certain disorders associated with fatty acid metabolism. Prolonged Parenteral nutrition may be necessary in patients suffering from a variety of gastrointestinal motility, malabsorptive, or metabolic disorders. As the number of patients relying on prolonged Parenteral nutrition support increases, associations between Parenteral nutrition and a group of hepatobiliary complications emerge. These Parenteral nutrition associated liver dysfunction varies according to age. ${ }^{31}$ Cholestatic syndromes occur more frequently with children, whereas steatosis and steatohepatitis are noted more commonly with adults. ${ }^{32}$

Clinical studies suggest that parenteral nutrition-related hepatic steatosis is primarily related to the effects of excess caloric intake, usually in the form of dextrose or glucose, and impaired hepatic secretion of TG. Increased hepatic fat deposition may begin with infusions of highly concentrated glucose and amino acids stimulating increased insulin secretion. Subsequent hyper insulinemia promotes lipogenesis and synthesis of TG from glucose while inhibiting mitochondrial fatty acid oxidation. ${ }^{31,33}$

\section{Disturbed liver function and secondary lipid affection}

The liver has remarkable capacity to recover from injury by regeneration. Such regenerative potential is essential for survival following partial hepatectomy (as in tumor removal or live-donor liver transplantation) and from acute and chronic liver injury secondary to toxins, infections, immune dysfunction, metabolic diseases, or other causes. ${ }^{34}$ Nevertheless, liver diseases remain an important cause of morbidity and mortality, and inadequate hepatic regeneration likely contributes. A number of studies have identified fatty liver as an important risk factor for impaired liver regeneration in humans..$^{35,36}$

Recent reports have characterized the decline in systemic adipose tissue stores and the consequent rise in circulating and hepatic FFA and specific amino acids that occur in response to partial hepatecto my prior to the onset of regeneration. ${ }^{37-39}$ Systemic adipose tissue depletion has also been observed in various models of toxin-induced liver regeneration. ${ }^{37}$ These findings together suggest that catabolism of systemic adipose tissue might regulate the hepatic regenerative response to surgical and toxin-induced loss of liver mass. ${ }^{40}$ It has long been recognized that the early regenerating liver transiently accumulates hepatocellular fat after partial hepatectomy. ${ }^{38,41}$

It has been suggested that the inhibitory effect of dextrose supplementation on liver regeneration might be secondary to the suppressive effect of such supplementation on the release of FFA from systemic adipose stores, and infusion of an inhibitor of $\beta$-oxidation, has been reported to impair regeneration. ${ }^{42}$ Moreover, Parenteral administration of lipid emulsions has been reported to accelerate $\mathrm{pH}-$ induced regeneration. ${ }^{43}$

On the other hand, chronic steatosis has been associated with adverse outcomes after major hepatic resection in humans. It has been shown that the risk of postoperative complications in patients with any degree of steatosis undergoing partial hepatectomy was double that of their non-steatotic counterparts, and that those with severe steatosis had an almost 3-fold increased risk of death. ${ }^{44}$ In addition, a study of patients undergoing liver resection for living-related liver donation showed reduced recovery of liver volume over the initial 3 months following surgery in patients with mild steatosis versus no steatosis. ${ }^{45}$

\section{Lipid profile in different diseases}

As the liver plays an essential role in lipid metabolism, several stages of lipid synthesis and transportation, it is reasonable to expect an abnormal lipid profile in those having liver dysfunction. There is prominent decline in plasma cholesterol and TG levels in patients with severe hepatitis and hepatic failure because of reduction of lipoprotein biosynthesis. ${ }^{46}$

Ghadir et al. ${ }^{47}$ reported that lower lipid levels are found in patients with liver diseases, and all the studied variables (HDL, LDL, total cholesterol and TG) were significantly lower in cirrhotic patients than in the comparison healthy normolipidemic group. Furthermore, the decrease in the serum HDL, LDL and total cholesterol (but not TG) had a positive correlation with the severity of liver damage.

The significant decline in the serum total cholesterol and TG levels in cirrhotic patients compared with healthy people has been confirmed earlier in other studies, which is reasonably expected since liver biosynthesis has been reduced. Mehbob et al. ${ }^{48}$ studied 160 patients with chronic liver diseases. They found a significant decline in the serum total cholesterol and TG levels of those patients. Siagris et al. ${ }^{49}$ performed a study on 155 patients infected with $\mathrm{HCV}$ and 138 healthy controls. They found that serum total cholesterol was lower in patients than the comparison group. In addition, Selimoglu et al. ${ }^{50}$ found that HDL levels were lower with more advanced disease. Kumar \& Harisha ${ }^{51}$ reported that, a significant difference was observed between patients and the comparison group in all lipid profile values studied and in severe liver disease as the liver function deteriorates, more decline is observed in LDL, HDL, total cholesterol levels and TG levels. Similar results were reported by Mandal et al. ${ }^{52}$ Subhan et al. ${ }^{53}$ and Varghese et al..$^{54}$ where they showed a progressive decline in the lipid levels with progression of liver disease.

Disorders of lipid metabolism in acute hepatitis are common. Plasma levels of total cholesterol, HDL were significantly lower in the patients at the acute phase of hepatitis than those in normal subjects, whereas plasma levels of TG and LDL were obviously higher in the patients than in normal subjects. Moreover, patients' plasma levels of total cholesterol, LDL and HDL were lower at the active phase of the diseases than at the recovering phase, which indicating that acute liver damage could significantly influence lipid metabolism. ${ }^{55}$

On the other hand, this was not in agreement with the finding reported by Zuberi \& Husnain ${ }^{56}$ where they found a significant elevation in serum cholesterol level in hepatitis than in cirrhosis and cancer. Serum TG and serum LDL were also significantly raised. Hyperlipidaemia in such patients could result from an inability of the liver to excrete lipids into the bile as a result of parenchymal damage. It is logical to conclude that this increase is initial in the course of the disease and only temporary. In later stages, the decline might be due to decreased synthesis in liver cells as cirrhosis involves considerable destruction of hepatic cells. ${ }^{57}$

\section{Fatty liver disease and atherogenic risk}

Elevated levels of blood lipids are well-documented risk factors for cardiovascular disease. Cardiovascular disease remains the leading cause of death in the adult population, and it has been shown that the atherosclerotic process begins in childhood. ${ }^{58,59}$ In children, investigators found that the extent to which the intimal surface was covered with atherosclerotic lesions was significantly associated with elevation of concentrations of total cholesterol, LDL, TG, and lower concentration of HDL. Ratios of cholesterol ester-rich lipoprotein levels (total cholesterol/HDL and LDL/HDL) are well-established predictors of cardiovascular disease. ${ }^{59}$ More recently, the TG/HDL ratio has been shown to be a strong predictor of cardiovascular disease. ${ }^{60,61}$ 
Nobili et al. ${ }^{62}$ described the link between severity of liver disease and atherogenic risk in children with NAFLD. The results show that the degree of liver injury and stage of fibrosis positively correlates with established markers of atherogenic risk. The association between liver damage and an atherogenic profile is independent of obesity, insulin resistance, and the presence of metabolic syndrome in children with NAFLD. The atherogenic profile is particularly prominent in patients with established NASH as compared to those with simple steatosis. Taken together, these results suggest a significant effect of the severity of NAFLD on cardiovascular risk in children.

The pathogenic mechanisms leading the progression of atherosclerotic lesions are various and involve endothelial cells, inflammatory cells, and platelets. Clinical observations indicated that NAFLD might be an independent risk factor for coronary artery disease. Moreover, NASH may increase atherosclerotic and cardiovascular risks by local overexpression of inflammatory mediators and endothelial damage. These aspects suggest the necessity for an early therapeutic intervention in NASH patients, not only for ameliorating the liver injury, but also for improving the systemic proatherogenic state. ${ }^{63,64}$

\section{Dietary restriction or fasting and lipid metabolism}

Caloric restriction has been shown to increase permanence, improve health and expand lifespan, reduces the morbidity, including autoimmune diseases, atherosclerosis, cardiomyopathies, cancer, diabetes, renal diseases, and respiratory diseases ${ }^{65-67}$ Weight loss with a low-fat diet decreases the plasma LDL concentration by increasing the catabolism of LDL. Weight loss also delays the catabolism of HDL with a concomitant reduction in the secretion of HDL with a net result of maintained HDL levels. ${ }^{68}$

Fasting is demarcated as a partial or total refraining from all foods, or a select abstention from prohibited foods. Fasting has been the subject of abundant scientific soundings. ${ }^{69}$ According to the Islamic Hijri calendar, Ramadan is the holiest month and Muslims fast during this month. It lasts between 29 to 30days. Believers are commanded to abstain from food, drink and conjugal relationships from sunrise to sunset.

Many physiological and psychological changes take place during Ramadan. Some studies in the eastern Mediterranean area have shown an increase of HDL concentration during Ramadan fast. ${ }^{70,71}$ A balanced diet, even less in quantity than normal, is sufficient to keep a person healthy and active during Ramadan fast. Lipid profile is affected by dietary habit, amount and type of fat present in the daily diet, amount of simple sugar intake and exercise performed. ${ }^{72,73}$ Some studies showed positive effects of fasting on the lipid profile changing them in anti-atherogenic direction. ${ }^{74-77}$

A significant reduction of serum total cholesterol, LDL and a significant rise in serum HDL were observed in the present study during one month of Ramadan fasting but serum TG showed no significant difference. The reduction of serum total cholesterol at the end of one month Ramadan fast was $8.31 \%{ }^{77}$ This is in agreement with the study done by Al Hourani et al. ${ }^{78}$ These studies, suggested that fasting may be beneficial to health and have some protective role against the development of atherosclerotic cardiovascular disease.

Shah et al. ${ }^{79}$ found that blood glucose showed significant reduction post Ramadan fasting. Serum cholesterol, TG and LDL showed reduction; however reduction was not significant in case of TG levels.
Serum HDL showed significant elevation after Ramadan fasting. This finding is supported by Attarzadeh. ${ }^{80}$ However; another study reported a decrease in HDL. ${ }^{81}$

Shehab et al. ${ }^{82}$ observed significant improvements in HDL, and LDL levels even after four weeks post Ramadan. Ramadan-like fasting may be considered for more effective lipid and lipoprotein control. ${ }^{82}$ Generally, low caloric intake and dietary restriction have a positive impact on lowering body weight, blood glucose, glycosylated hemoglobin, TG, total cholesterol, and LDL. There are also positive changes in LDL subclass distribution and a similar shift in HDL subclass distribution. ${ }^{83}$

\section{Conclusion}

The liver plays a key role in lipid metabolism. It largely affects fatty acid synthesis and lipid circulation through lipoprotein synthesis. Eventually the accumulation of lipid droplets into the hepatocytes results in hepatic steatosis, which may develop as a consequence of multiple dysfunctions such as alterations in $\beta$-oxidation, very low density lipoprotein secretion, and pathways involved in the synthesis of fatty acids. In addition an increased circulating fatty acid may also to be a major determinant in the pathogenesis fatty liver disease. Hepatic lipid metabolism is a highly coordinated process, and liver disease will certainly have some compromise on lipid homeostasis leading to pathological consequences. Manipulating lipid metabolism by changing caloric intake and diet restriction may have positive impact on health and better quality of life. ${ }^{84-86}$

\section{Acknowledgements}

None.

\section{Conflict of interest}

Author declares that there is no conflict of interest.

\section{References}

1. Aballay LR, Eynard AR, Diaz Mdel P, et al. Overweight and obesity: a review of their relationship to metabolic syndrome, cardiovascular disease, and cancer in South America. Nutr Rev. 2013;71(3):168-179.

2. Bedpogni G, Bellentani S. Fatty liver: how frequent is it and why? Ann Hepatol. 2004;3(2):63-65.

3. Labrecque DR, Abbas Z, Anania F, et al. World Gastroenterology Organization global guidelines: Nonalcoholic fatty liver disease and nonalcoholic steatohepatitis. J Clin Gastroenterol. 2014;48(6):467-473.

4. Machado M, Marques-Vidal P, Cortez-Pinto H. Hepatic histology in obese patients undergoing bariatric surgery. $J$ Hepatol. 2006;45(4):600-606.

5. Bahrami H. Non-alcoholic fatty liver disease in developing countries. World J Gastroenterol. 2005;11(24):3808-3809.

6. Ray K. NAFLD-the next global epidemic. Nat Rev Gastroenterol Hepatol. 2013;10(11):621.

7. Loomba R, Sanyal AJ. The global NAFLD epidemic. Nat Rev Gastroenterol Hepatol. 2013;10(11):686-690.

8. Abumrad NA, Davidson NO. Role of the gut in lipid homeostasis. Physiol Rev. 2012;92(3):1061-1085.

9. Hoover-Plow J, Huang M. Lipoprotein(a) metabolism: potential sites for therapeutic targets. Metabolism. 2013;62(4):479-491.

10. Rui L. Energy metabolism in the liver. Compr Physiol.2014;4(1):177-197. 
11. Reddy JK, Rao MS. Lipid metabolism and liver inflammation. II. Fatty liver disease and fatty acid oxidation. Am J Physiol Gastrointest Liver Physiol. 2006;290(5):G852-G858.

12. Reddy JK, Hashimoto T. Peroxisomal beta-oxidation and peroxisome proliferator-activated receptor alpha: an adaptive metabolic system Annu Rev Nutr. 2001;21:193-21230.

13. Wanders RJA, Ruiter JPN, Ijlst L, et al. The enzymology of mitochondrial fatty acid beta-oxidation and its application to followup analysis of positive neonatal screening results. J Inherit Metab Dis. 2010;33(5):479-494.

14. Rao MS, Reddy JK. PPARalpha in the pathogenesis of fatty liver disease. Hepatology. 2004;40(4):783-786.

15. Poirier Y, Antonenkov VD, Glumoff T, et al. Peroxisomal $\beta$-oxidation - A metabolic pathway with multiple functions. Biochim Biophys Acta. 2006;1763(12):1413-1426.

16. Chalasani N, Younossi Z, Lavine JE, et al. The diagnosis and management of non-alcoholic fatty liver disease: practice Guideline by the American Association for the Study of Liver Diseases, American College of Gastroenterology, and the American Gastroenterological Association. Hepatology. 2012;55(6):2005-2023.

17. Bradbury MW. Lipid metabolism and liver inflammation. I. Hepatic fatty acid uptake: possible role in steatosis. Am J Physiol Gastrointest Liver Physiol. 2006;290(2):G194-G198.

18. Browning JD, Horton JD. Molecular mediators of hepatic steatosis and liver injury. J Clin Invest. 2004;114(2):147-152.

19. Evans RM, Barish GD, Wang YX. PPARs and the complex journey to obesity. Nat Med. 2004;10(4):355-361.

20. Fabbrini E, Magkos F. Hepatic Steatosis as a Marker of Metabolic Dysfunction. Nutrients. 2015;7(6):4995-5019.

21. Nellemann B, Gormsen LC, Christiansen JS, et al. Post-absorptive VLDL-TG Fatty Acid Storage in Adipose Tissue in Lean and Obese Women. Obesity. 2010;18(7):1304-1311.

22. Ebbert JO, Jensen MD. Fat Depots, Free Fatty Acids, and Dyslipidemia Nutrients. 2013;5(2):498-508.

23. Mollard RC, Senechal M, Macintosh AC, et al. Dietary determinants of hepatic steatosis and visceral adiposity in overweight and obese youth at risk of type 2 diabetes. Am J Clin Nutr. 2014;99(4):804-812.

24. Hamaguchi M, Kojima T, Takeda N, et al. The metabolic syndrome as a predictor of nonalcoholic fatty liver disease. Ann Intern Med. 2005;143(10):722-728.

25. Adams LA, Lymp JF, St Sauver J, et al. The natural history of nonalcoholic fatty liver disease: a population-based cohort study. Gastroenterology. 2005;129(1):113-121.

26. Browning JD, Szczepaniak LS, Dobbins R, et al. Prevalence of hepatic steatosis in an urban population in the United States: impact of ethnicity. Hepatology. 2004;40(6):1387-1395.

27. Crabb DW, Galli A, Fischer M, et al. Molecular mechanisms of alcoholic fatty liver: role of peroxisome proliferator-activated receptor alpha. Alcohol. 2004;34(1):35-38.

28. Wieckowska A, Feldstein AE. Nonalcoholic fatty liver disease in the pediatric population: a review. Curr Opin Pediatr. 2005;17(5):636-641.

29. Schwimmer JB, Deutsch R, Kahen T, et al. Prevalence of fatty liver in children and adolescents. Pediatrics. 2006;118(4):1388-1393.

30. Scaglioni F, Ciccia S, Marino M, et al. ASH and NASH. Dig Dis. 2011;29(2):202-210.
31. Lee V. Liver Dysfunction Associated with Parenteral Nutrition: What Are the Options? Practical Gastroenterology. 2006;30(12):49-68.

32. Kumar Jain A, Teckman JH. Newly Identified Mechanisms of Total Parenteral Nutrition Related Liver Injury. Advances in Hepatology. 2014;621380:1-7.

33. Kelly DA. Preventing parenteral nutrition liver disease. Early Hum Dev. 2010;86(11):683-687.

34. Michalopoulos GK. Liver regeneration. $J$ Cell Physiol. 2007;213(2):286-300.

35. Kele PG, Vander Jagt EJ, Gouw AS, et al. The impact of hepatic steatosis on liver regeneration after partial hepatectomy. Liver Int. 2013;33(3):469-475.

36. Hamano M, Ezaki H, Kiso S, et al. Lipid overloading during liver regeneration causes delayed hepatocyte DNA replication by increasing ER stress in mice with simple hepatic steatosis. $J$ Gastroenterol. 2014;49(2):305-316.

37. Gazit V, Weymann A, Hartman E, et al. Liver regeneration is impaired in lipodystrophic fatty liver dystrophy mice. Hepatology. 2010;52(6):2109-2117.

38. Newberry EP, Kennedy SM, Xie Y, et al. Altered hepatic triglyceride content after partial hepatectomy without impaired liver regeneration in multiple murine genetic models. Hepatology. 2008;48(4):1097-1105.

39. Rudnick DA, Dietzen DJ, Turmelle YP, et al. Serum alpha-NH-butyric acid may predict spontaneous survival in pediatric acute liver failure. Pediatr Transplant. 2009;13(2):223-230.

40. Rundnick DA, Davidson NO. Functional Relationships between Lipid Metabolism and Liver Regeneration. Int J Hepatol. 2012;549241:1-8.

41. Domitrovic R, Milin C, Radosevic-Stasic B. Liver fatty acid and element changes after partial hepatectomy in mice fed olive oil- and corn oilenriched diets. Biol Trace Elem Res. 2006;109(1):61-74.

42. Weymann A, Hartman E, Gazit V, et al. p21 is required for dextrosemediated inhibition of mouse liver regeneration. Hepatology. 2009;50(1):207-215.

43. Kin SY, Kim D, Chol J, et al. Lipids in Parenteral Nutrition on Liver Regeneration After Massive Liver Resection in Rats. J Gastroenterol Hepatol Res. 2014;3(9).

44. De Meijer VE, Kalish BT, Puder M, et al. Systematic review and metaanalysis of steatosis as a risk factor in major hepatic resection. Br J Surg. 2010;97(9):1331-1339.

45. Cho JY, Suh KS, Kwon CH, et al. Mild hepatic steatosis is not a major risk factor for hepatectomy and regenerative power is not impaired. Surgery. 2006;139(4):508-515.

46. Halsted $\mathrm{CH}$. Nutrition and alcoholic liver disease. Semin Liver Dis 2004;24(3):289-304

47. Ghadir MR, Riahin AA, Havaspour A, et al. The relationship between lipid profile and severity of liver damage in cirrhotic patients. Hepat Mon. 2010;10(4):285-288.

48. Mehboob F, Ranjha FA, Masud S. Changes in Serum Lipid Profile Among Patients Suffering From Chronic Liver Disease. Annals. 2007;13(3):209-211.

49. Siagris D, Christofidou M, Theocharis GJ, et al. Serum lipid pattern in chronic hepatitis C: histological and virological correlations. J Viral Hepat. 2006;13(1):56-61.

50. Selimoglu MA, Aydogdu S, Yagci RV. Lipid parameters in childhood cirrhosis and chronic liver disease. Pediatr Int. 2002;44(4):400-403. 
51. Kumar WMR, Harisha E. Assessment of lipid profile changes with respect to severity of liver dysfunction in cirrhosis of liver. Indian Journal of Basic and Applied Medical Research. 2015;4(2):56-63.

52. Mandal SK, Sil K, Chatterjee S, et al. A Study on Lipid Profiles in Chronic Liver Diseases. Natl J Med Res. 2013;3(1):70-72.

53. Subhan F, Khan I, Arif R, et al. Serum lipid profile as an indicator of the severity of liver damage in cirrhotic patients. Rawal Medical Journal. 2012;37(4):387-389.

54. Varghese JS, Krishanaprasad K, Upadhuyay R, et al. Lipoprotein profile in cirrhosis of liver. Eur J Gastroenterol Hepatol. 2007;19(6):521-522.

55. Luo $\mathrm{L}, \mathrm{Pu} \mathrm{X}$, Wang $\mathrm{Y}$, et al. Impaired plasma lipid profiles in acute hepatitis. Lipids in Health and Disease. 2010;9:5.

56. Rahaam A, Zuberi SJ, Husnain SN. Lipid Pattern in Liver Disease. J Pak Med Assoc. 1982;32(12):275-278.

57. Remaley ET, Rifai N, Warnick GR. Lipids, lipoproteins, apolipoprotiens and other Cardiac risk factors. In: Burtis CA, Bruns DE, editors. Tietz Fundamentals of Clinical Chemistry and Molecular Diagnostics. USA 2015. p. 388-409.

58. Daniels SR, Pratt CA, Hayman LL. Reduction of Risk for Cardiovascular Disease in Children and Adolescents. Circulation. 2011;124(15):1673-1686.

59. Pahkala K, Hietalampi H, Laitinen TT, et al. Ideal Cardiovascular Health in Adolescence: Effect of Lifestyle Intervention and Association with Vascular intima-Media Thickness and Elasticity (The Special Turku Coronary Risk Factor Intervention Project for Children [STRIP] Study). Circulation. 2013;127(21):2088-2096.

60. Mclaughlin T, Reaven G, Abbasi F, et al. Is there a simple way to identify insulin-resistant individuals at increased risk of cardiovascular disease? Am J Cardiol. 2005;96(3):399-404.

61. Quijada Z, Paoli M, Zerpa Y, et al. The triglyceride/HDL-cholesterol ratio as a marker of cardiovascular risk in obese children; association with traditional and emergent risk factors. Pediatr Diabetes. 2008;9(5):464-471.

62. Nobili V, Alkouri N, Bartuli A, et al. Severity of liver injury and atherogenic lipid profile in children with nonalcoholic fatty liver disease. Pediatr Res. 2010;67(6):665-670.

63. Gaudio E, Nobili V, Franchito A, et al. Non-alcoholic fatty liver disease and atherosclerosis. Intern Emerg Med. 2012;7(Suppl 3):S297-S305.

64. Kuchukazman M, Ata N, Yavuz B, et al. Evaluation of early atherosclerosis markers in patients with nonalcoholic fatty liver disease. Eur J Gastroenterol Hepatol. 2013;25(2):147-151.

65. Imai S. SIRT1 and caloric restriction: an insight into possible tradeoffs between robustness and frailty. Curr Opin Clin Nutr Metab Care. 2009; 12(4):350-356.

66. Vaquero A, Reinberg D. Calorie restriction and the exercise of chromatin. Genes Dev. 2009;23(16):1849-1869.

67. Sathananthan M, Shah M, Edens KL, et al. Six and 12 Weeks of Caloric Restriction Increases beta Cell Function and Lowers Fasting and Postprandial Glucose Concentrations in People with Type 2 Diabetes. $J$ Nutr. 2015;149(9):2046-2051.

68. Ng TWK, Watts GF, Barrett PHR, et al. Effect of Weight Loss on LDL and HDL Kinetics in the Metabolic Syndrome: Associations with changes in plasma retinol-binding protein-4 and adiponectin levels. Diabetes Care. 2007;30(11):2945-2950.
69. Hadikhafaji HAR, Al Suwaidi JM. Ramadan fasting and lipid profile. In: Chtourou H, editor. Effects of Ramadan Fasting on Health and Athletic Performance. USA; 2013. p. 1-13.

70. Khaled BM, Bendahmane M, Belbraouet S. Ramadan fasting induces modifications of certain serum components in obese women with type 2 diabetes. Saudi Med J. 2006;27(1):23-26.

71. Khatib FA, Shafagoj YA. Metabolic alterations as a result of Ramadan fasting in non-insulin-dependent diabetes mellitus patients in relation to food intake. Saudi Med J. 2004;25(12):1858-1863.

72. Tsai AC, Sandretto A, Chung YC. Dieting is more effective in reducing weight but exercise is more effective in reducing fat during the early phase of a weight-reducing program in healthy humans. J Nutr Biochem. 2003;14(9):541-549.

73. Afrasiabi A, Hassanzadeh S, Sattarivand R, et al. Effects of Ramadan fasting on serum lipid profiles on 2 hyperlipidemic groups with or without diet pattern. Saudi Med J. 2003;24(1):23-26.

74. Lamine F, Bouguerra R, Jabrane J, et al. Food intake and high density lipoprotein cholesterol levels changes during ramadan fasting in healthy young subjects. Tunis Med. 2006;84(10):647-650.

75. Adlouni A, Ghalim N, Benslimane A, et al. Fasting during Ramadan induces a marked increase in high-density lipoprotein cholesterol and decrease in low-density lipoprotein cholesterol. Ann Nutr Metab. 1997;41(4):242-249.

76. Saleh SA, El-Kemery TA, Farrag KA, et al. Ramadan fasting: relation to atherogenic risk among obese Muslims. J Egypt Public Health Assoc; 2004;79(5-6):461-483.

77. Akhtaruzzaman M, Hoque N, Choudhury MBK, et al. Effect of Ramadan Fasting on Serum Lipid Profile of Bangladeshi Female Volunteers. Bangladesh Journal of Medical Biochemistry. 2015;7(2):47-51.

78. Al Hourani HM, Atoum MF, Akel S, et al. Effects of Ramadan Fasting on Some Hematological and Biochemical Parameters. Jordan Journal of Biological Sciences. 2009;2(3):103-108.

79. Shah S, Kumar GA, Singh SB, et al. Effect of Ramadan fasting on blood sugar level and lipid profile in healthy Meos. Trans world Medical Journal. 2014;2(1):1-5.

80. Attarzadeh Hosseini SR, Sardar MA, Hejazi K, et al. The Effect of Ramadan Fasting and Physical Activity on Body Composition, Serum Osmolarity Levels and Some Parameters of Electrolytes in Females. Int J Endocrinol Metabol. 2013;11(2):88-94.

81. Mauzzam MG. Ramadan fasting and medical science. Bangladesh Journal of Medical Science 1991;3(1):8-15.

82. Shehab A, Abdulle A, El Issa A, et al. Favorable Changes in Lipid Profile: The Effects of Fasting after Ramadan. PLoS ONE. 2012;7(10):e47615.

83. Harder H, Dinese B, Astrup A. The effect of a rapid weight loss on lipid profile and glycemic control in obese type 2 diabetic patients. Int J Obes Relat Metab Disord. 2004;28(1):180-182.

84. Trepanowski JF, Canale RE, Marshall KE, et al. Impact of caloric and dietary restriction regimens on markers of health and longevity in humans and animals: a summary of available findings. Nutr J. 2011;10:107.

85. Weiss EP, Fontana L. Caloric restriction: powerful protection for the aging heart and vasculature. Am J Physiol Heart Circ Physiol. 2011;301(4):1205-1219.

86. Fox SI. The Digestive System Human Physiology. New York, USA McGraw-Hill; 2016. p. 619-660. 